systems, demand time, research and trial for their effective resolution. A new polymeric flocculant known as 'Primafloe $C .7$ ' is now available in commercial quantities for such purposes and a brief account of its constitution, merits, and properties is to bo found in a short article entitled, "A Solution to Waste-handling Problems" (Rohm and Haas Reporter, Rohm and Haas Co., Philadelphia (London, Lennig Chemicals, Ltd., 26-28, Bedford Row), 23, 5, September-October 1965). This new product is a water-soluble cationic polyelectrolyte, supplied as a free-flowing, slightly hygroscopic powder, one of the latest in a series of such compounds to be offered by Rohm and Haas. It readily dissolves in water at a concentration of 2-6 per cent; before use, this solution is normally diluted to a working concentration of about $0 \cdot 1$ per cent. It is claimed that, in the clarification of turbid waters, 'Primafloc $C-7$ ' can be used as a prime coagulant; addition of inorganic coagulants such as alum or ferric sulphate is unnecessary. Furthermore, addition of 1-10 p.p.m. of this compound, together with a suitable clay, will rapidly and effectively remove suspended colids, an advantage where restrictions preclude the use of inorganic coagulants. If inorganic coagulants are used, 'Primafloc $C-7$ ' $(0 \cdot 1-$ I p.p.m.) can greatly increase floc growth and settling rates, thus improving final water clarity. Since most naturally occurring suspended particles have a negative surface charge, causing them to repel one another and remain suspended, 'Primafloc' cationic polyelectrolytes, containing as they do a larger number of positively charged sites, work on the principle of neutralizing these negative charges, causing particles to coagulate, and thus to settle. There are other 'Primafloc' polyelectrolytes available for various purposes in waste-handling problems.

\section{Procter and Gamble, Ltd.}

THe internationally known Procter and Gamble organization comprises not only the parent and subsidiary companies in the United States, but also companies operating in Belgium, Canada, France, Great Britain, Italy, Mexico, Peru, Philippines, Switzerland, Venezuela, and West Germany. The principal products manufactured by this group are the familiar retail brands of synthetic detergents, edible shortenings and margarines for the bakery trade, industrial soaps, textile brands of soap in various forms, certain building trade specialities, and other products including refined and hardened edible oils, fatty acids, glycerine, fat additives for animal feeds, etc. All these products are manufactured and sold in Great Britain by Procter and Gamble, Ltd., whose financial statement for the year ended June 30, 1965, has been published (Procter and Gamble, Ltd., Financial Statement for the year ended 30th June 1965. Pp. 16. Newcastle upon Tyne: Procter and Gamble, Ltd., 1965). Apart from the commendably lucid statement of the financial aspects of this company's affairs, which has previously been commented on in these columns (Nature, 204, $1257 ; 1964)$, this report contains much of both technical and economic interest. The emphasis in this report is on the functions and activities of those members of the Company whose task it is to buy the raw materials, goods and services needed in the course of day-to-day operations. Under the heading "Buying Better Value for Consumers" are discussed now products and new processes, the buyer's broad responsibilities, product improvement, transport and international operations. Trensport is particularly emphasized in this brochure, which contains another series of most pleasing illustrations in colour of buying and shipping activities, the work of David Knight, who contributed so much to the artistic value of the 1964 statement. On the research side, a new extension of the Longbenton research laboratories has made it possible for all major research departments to be brought under one roof. The job of the Research Division is to ensure that every product is formulated to give maximum performance and appeal in its own field. One instance of this may be quoted: in the past 16 years, the Division's home laundry carried out the equivalent of 80,000 weekly washes, equal to 1,600 years of domestic washing experience for one household; its controlled test methods are based on present-day domestic washing habits as established by consumer research. It is noted that during the year under review, the Company has co-operated fully with the Monopolies Commission in their investigation of the household detergent industry.

\section{Soils and Foundations}

Movement resulting from interactions between soils and foundations was the subject of a recent publication by the Building Research Station, a note of which appeared in Nature $(208,1153 ; 1965)$. A further publication in the same series outlines the soil and other investigations necessary in assessing the suitability of sites for new buildings and is entitled. Soils and Foundations: 2 (Building Research Station Digest (Second Series), No. 64. Pp. 6. London, H.M.S.O., 1965. 4d.). The need for thorough site investigation when any building work is envisaged which may be potentially vulnerable to conditions existing below ground-level is a prior charge on the project, and cannot be too strongly emphasized; this applies equally to alterations and extensions of existing work as to entirely new schemes. The comparative scarcity of building sites available for large projects, especially in built-up industrialized areas, has led to the use of sites which may have been avoided in the past for one reason or another but have now come up for serious reconsideration; in such circumstances early re-appraisal of the site is imperative, so that any special measures that may be required to deal with difficult conditions can be planned at the outset. This publication outlines the main stages to be undertaken in any investigation of site conditions; these are, first, information available off site (appeal to the local authority, Ordnance Survey maps and records from local sources for knowledge of soil and general conditions, for example, infilled ponds, ditches, streams and disused pipes). Secondly, it is necessary to undertake site reconnaissance, which should include topography, possibility of flooding, ground water-level and vegetation as a clue to the nature of sub-soil. The third stage embraces site history, whether virgin land or a previously used site. The fourth covers soil investigations on site, which means those concerned with relatively simple observations or tests, and, where necessary, full-scale soil mechanies to determine the strengths and deformation. characteristics of the soil under load and to identify conditions in which soils are otherwise susceptible to movement. Soils and Foundations : 2 contains a useful table of soil identification, a summary of the scope of site investigations both in plan and in depth, details of information required from any one site, and notes on sampling (use of post-hole auger, etc.). An appendix on various sources of information about any particular area concludes what is undoubtedly a most valuable guide to an often complex problem of site investigation.

\section{Bibliography on Religion and Science}

A BIBLIOGRAPHY on religion and science, compiled by A. Maltby (The Library Association. Special Subject List No. 46: Religion and Science. Pp. 36. London: The Library Association, 1965 . 10s.; L.A. members, $7 s .6 d$.$) is arranged in three sections covering, respectively,$ the history of the conflict between religion and science prior to the nineteenth century; the nineteenth century struggle; and the relation between religion and science in the twentieth century. Nearly 200 of the 238 entries, some of which are annotated, fall into the third section. There is an index of authors. Although the bibliography appears to be representative, the basis of selection is not stated and there is one startling omission: A. N. Whitehead. 